\title{
Adults with Down syndrome have reduced cardiac response after light exercise testing
}

\author{
J. C. Vis • H. A. C. M. De Bruin-Bon • B. J. Bouma • \\ S. A. Huisman • L. Imschoot $•$ K. van den Brink • \\ B. J. M. Mulder
}

Published online: 14 February 2012

(C) The Author(s) 2012. This article is published with open access at Springerlink.com

\begin{abstract}
Objective Physical fitness is reduced in adults with Down syndrome (DS). The present study was conducted to elucidate the exercise response in adults with DS.

Design Case controlled before-after trial.

Setting Residential centre for people with intellectual disabilities.

Participants 96 Adults with DS, 25 non-DS adults with an intellectual disability, 33 controls.

Interventions Echocardiography to exclude heart defects and to measure cardiac index (CI) in the supine position, supine position with raised legs, and following ten knee bends.

Main outcome measure Exercise testing

Results At rest, mean CI was not significantly different between persons with DS and controls (2.3 vs. $\left.2.41 / \mathrm{min} / \mathrm{m}^{2}, p=0.3\right)$.
\end{abstract}

J. C. Vis • H. A. C. M. De Bruin-Bon • B. J. Bouma •

B. J. M. Mulder $(\square)$

Department of Cardiology, Academic Medical Centre,

Meibergdreef 9,

1105 AZ Amsterdam, the Netherlands

e-mail: b.j.mulder@amc.uva.nl

\section{S. A. Huisman}

Prinsenstichting,

Residential Centre for People with Intellectual Disabilities, postbus 123 ,

1440 AC Purmerend, the Netherlands

L. Imschoot

ASVZ, Residential Centre for People with Intellectual Disabilities,

Postbus 121,

3360 AC Sliedrecht, the Netherlands

K. van den Brink

's Heeren Loo,

Residential Centre for People with Intellectual Disabilities,

Postbus 10352680 BA Monster, the Netherlands
However, mean CI after exercise was significantly lower in DS ( 2.9 vs. $\left.3.7 \mathrm{l} / \mathrm{min} / \mathrm{m}^{2}, p<0.001\right)$ and mean CI increase from rest to exercise was more than $50 \%$ lower in DS. On the contrary, CI after exercise was similar among controls and non-DS adults with an intellectual disability. Significantly lower stroke volumes in DS were found with insufficient heart rate response.

Conclusions $\mathrm{CI}$ at rest was similar in adults with DS and controls; however persons with DS have a diminished cardiac response to exercise. Stroke volumes were significantly lower in DS during exercise and a compensated heightened heart rate was absent.

Keywords Down syndrome · Cardiac response · Cardiac index $\cdot$ Exercise testing $\cdot$ Echocardiography $\cdot$ Stroke volume

\section{Introduction}

Average life expectancy of adults with Down syndrome (DS) has increased from a mere 12 years in the 1940 s to 60 years in present-day populations [1-4]. This is due to improved techniques and outcome of corrective cardiac surgery and available advanced therapies for DS patients $[5,6]$. However, DS is associated with a high prevalence of comorbid conditions, such as osteoarthritis, obesity and Alzheimer's disease at relatively young age [7-11]. Therefore there has been growing attention for the prevention of conditions that lead to participation limitations and inactivity.

Lower levels of cardiovascular fitness have been reported previously in children and adults with DS compared with subjects without DS with or without an intellectual disability [12-14]. Also aerobic capacity exhibits a different age-related response in persons with DS as relative $\mathrm{VO} 2$ peak does not decline with age [15]. A combination of chronotropic 
incompetence $[16,17]$ and sympathetic dysfunction [18] may explain the very low maximal heart rate and could contribute to the reduction in aerobic power in persons with DS. Hypothetically, exercise programs could have a positive affect on overall health and lead to an increased quality of life in adults with DS. Most training programs among adults with DS did not yield the desired response of improved cardiovascular capacity [19, 20], although Tsimaras et al. found improvement of peak aerobic capacity in 25 adults with DS following a jog-walk training program [21]. We hypothesised that cardiac response to exercise would be lower in patients with DS. The primary goal of this study was to further elucidate the physiological response to physical activity in healthy adults with DS.

\section{Methods}

\section{Study population}

We included adults with an intellectual disability with and without DS, living in health care institutions (group homes) for people with intellectual disabilities. A control group of healthy non-athletic volunteers was randomly sampled from the Academic Medical Centre in Amsterdam (including hospital employees and medical students). Exclusion criteria were: 1) congenital heart defects 2) Alzheimer's disease 3) contraindications to exercise or physical incompetence to perform the exercise protocol (wheelchair user, orthopaedic injury) 4) unregulated thyroid function 5) patients without sinus rhythm. An electrocardiogram was taken to examine arrhythmias and thyroid function was checked in the participants with DS by medical charts according to the latest laboratory testing within 1 year. Approval was obtained from ethics boards of all participating institutions and informed consent was acquired from all subjects and/or their legal guardians

\section{Echocardiography}

An echocardiogram was performed in all adult patients with a portable GE VIVID I (Horten, Norway), by an experienced ultrasound technician and evaluated by a cardiologist. Echocardiography was performed to exclude congenital heart defects and standard echo measures were obtained in the supine position, supine position with raised legs (in $45^{\circ}$ angle to increase preload), and in the supine position following ten knee bends. All echocardiographic images were acquired according to recommendations of the American Society of Echocardiography $[22,23]$, recorded digitally, and analysed offline. The following echocardiographic dimensions were measured: left ventricular end-diastolic diameter (LVEDD), left ventricular outflow tract diameter ( $L V O T d)$, in parasternal short-axis view, velocity time integral $(V T I)$ in apical long- axis view by pulsed wave (PW) Doppler. The PW sample volume was positioned just proximal to the aortic valve leaflets, within the LVOT. The VTI was measured by tracing the leading edge of the velocity spectrum. Cardiac output was calculated using the following formulas: Area $(A)=(L V O T d / 2)^{2} \times \pi$; stroke volume $(S V)=A \times V T I$; $C O=S V \times$ heart rate. Cardiac output was adjusted for body surface area to obtain cardiac index (CI).

\section{Exercise test}

After echocardiographic measurements in supine position and in supine position with raised legs, patients had to perform a simple test. First, the patient was informed about the test procedure. Subsequently patients had to stand next to the examination bed and were asked to perform ten knee bends (without touching the legs or knees with the arms). Immediately after this task, patients had to lie on the examination bed in a supine position and echocardiographic measurements were repeated. The total duration of the task and the delay between the end of task and echocardiographic recordings were measured with a stopwatch. Task duration of less than $45 \mathrm{~s}$ was accepted. Patients who did not meet this criterion were excluded as their task performance was inadequate.

\section{Statistical analysis}

Descriptive statistics were used to describe baseline characteristics. Patient characteristics were compared between groups using chi-squared test in case of categorical variables whereas continuous variables were compared using oneway analysis of variance (ANOVA). Repeated measures ANOVA (Group X Condition) were applied to compare continuous variables among the three groups at rest, in a supine position with raised legs and after exercise. If Mauchly's test indicated that the assumption of sphericity had been violated, degrees of freedom were corrected using the Huynh-Feldt correction. Data are given as mean \pm standard deviation (SD) and the level of significance was set at $p<0.05$. A multiple linear regression model for cardiac index was done to adjust for baseline differences between groups. Those variables found to be significant by univariate analysis $(p<0.1)$ were put in the multivariate model. Statistical analysis was performed with the SPSS software for Windows XP version 16.0.

\section{Results}

Study population

In total, 96 adults with DS (mean age $42 \pm 11$ years, $48 \%$ males), 25 non-DS adults with an intellectual disability 
(mean age $50 \pm 11$ years, 60\% males) and 33 controls (mean age $40 \pm 11$ years, 55\% males) participated in this study. Baseline characteristics of all subject groups are shown in Table 1. Cardiac output testing at rest could be performed in all 154 subjects. However cardiac index after exercise could only be obtained from 52 DS patients, 11 non-DS adults with an intellectual disability and 22 controls (49\% of the included population). In the two groups with an intellectual disability, the patients with a severe cognitive impairment had a failed exercise test significantly more often (DS $p<0.001$ and non-DS $p=0.04$ ). All other baseline characteristics of patients with and without exercise testing were not significantly different, except for the non-DS adults with an intellectual disability; patients with a successful exercise test were on average $13 \mathrm{~kg}$ heavier. Mean time of exercise testing was $24 \pm 6 \mathrm{~s}$ and mean delay between exercise testing and echocardiographic recording was $32 \pm 13 \mathrm{~s}$. In total, 54 DS persons and 14 non-DS adults with an intellectual disability were excluded for exercise testing, because they were not able to execute the exercise test adequately or did not want to participate.

\section{Cardiac indices}

CI was not significantly different between adults with DS and controls at rest (2.3 vs $2.41 / \mathrm{min} / \mathrm{m}^{2}, p=0.3$ ) (Table 2). Even so, during increased preload, measured by raised legs ( 2.5 vs $2.7 \mathrm{l} / \mathrm{min} / \mathrm{m}^{2}, p=0.07$ ) cardiac index was similar.
However, CI after exercise was significantly lower in DS persons $\left(2.9\right.$ vs. $\left.3.7 \mathrm{l} / \mathrm{min} / \mathrm{m}^{2}, p<0.001\right)$. In controls, CI increased $69 \%$ from baseline to after exercise $(p<0.001)$. A lower CI increase of $30 \%$ was found in DS patients $(p<0.001)$. Heart rate response was comparable between DS persons and controls; however, a significantly lower stroke volume was observed in DS persons $(p<0.001)$, as shown in Fig. 1. Mean cardiac indices of all three groups after upright tilting of the legs and exercise are shown in Fig. 2. LVEDD was $40.1 \pm 4.4 \mathrm{~mm}$ in DS persons, $42.9 \pm 4.5 \mathrm{~mm}$ in non-DS adults with an intellectual disability and $50.0 \pm 3.9 \mathrm{~mm}$ in controls $(p<0.001)$. By multivariate analysis, independent predictors for absolute increase of cardiac index after exercise were age $(\beta=-0.21, p=0.009)$, left ventricular end-diastolic diameter $(\beta=0.33, p=0.009)$ and DS $(\beta=-0.46, p=0.001)$ (Table 3).

\section{Discussion}

The main finding of this study is that in healthy adults with DS, an increase in cardiac index is diminished after relatively light exercise test compared with healthy controls and non-DS adults with an intellectual disability. Mean increase of cardiac index after a simple exercise task was 50\% lower in individuals with DS compared with healthy controls. This diminished cardiac response after exercise was related to a lower mean stroke volume in DS as no group differences for heart rate

Table 1 Baseline characteristics

Values expressed as mean $\pm \mathrm{SD}$, $B M I$ body mass index, $B S A$ body surface area, $N A$ not applicable, $N S$ non significant

${ }^{\mathrm{a} C}$ Control versus non-Down syndrome patients with intellectual disability

${ }^{\mathrm{b}}$ Down syndrome patients versus non-Down syndrome patients with intellectual disability

${ }^{\mathrm{c} C o n t r o l ~ v e r s u s ~ D o w n ~ s y n d r o m e ~}$ patients

${ }^{\mathrm{d}}$ Criterium blood pressure $>140$ /

$90 \mathrm{mmHg}$

${ }^{\mathrm{e}}$ Criterium $\mathrm{BMI}>30 \mathrm{~kg} / \mathrm{m}^{2}$

\begin{tabular}{|c|c|c|c|c|}
\hline & \multicolumn{2}{|c|}{ Intellectual disability } & \multirow{2}{*}{$\begin{array}{l}\text { Controls } \\
n=33\end{array}$} & \multirow[t]{2}{*}{$p$-value } \\
\hline & $\begin{array}{l}\text { Down syndrome } \\
n=96\end{array}$ & $\begin{array}{l}\text { Non-Down-syndrome } \\
n=25\end{array}$ & & \\
\hline Male gender $(\%)$ & 48 & 60 & 55 & NS \\
\hline Age, $y r s$ & $42 \pm 11$ & $50 \pm 11$ & $40 \pm 11$ & $0.002^{\mathrm{a}, \mathrm{b}}$ \\
\hline Weight, $k g$ & $64 \pm 11$ & $77 \pm 15$ & $72 \pm 12$ & $<0.001^{\mathrm{b}, \mathrm{c}}$ \\
\hline Height, $\mathrm{cm}$ & $153 \pm 8$ & $168 \pm 13$ & $177 \pm 9$ & $<0.001^{\mathrm{a}, \mathrm{b}, \mathrm{c}}$ \\
\hline BMI, $\mathrm{kg} / \mathrm{m}^{2}$ & $27 \pm 4$ & $27 \pm 4$ & $23 \pm 3$ & $<0.001^{\mathrm{a}, \mathrm{c}}$ \\
\hline BSA, $m^{2}$ & $1.6 \pm 0.2$ & $1.9 \pm 0.2$ & $1.9 \pm 0.2$ & $<0.001^{\mathrm{b}, \mathrm{c}}$ \\
\hline Systolic blood pressure, $\mathrm{mm} \mathrm{Hg}$ & $115 \pm 13$ & $127 \pm 12$ & $115 \pm 11$ & $<0.001^{\mathrm{a}, \mathrm{b}}$ \\
\hline $\begin{array}{l}\text { Diastolic blood pressure, } \mathrm{mm} \mathrm{Hg} \\
\text { Intellectual disability }\end{array}$ & $73 \pm 8$ & $81 \pm 11$ & $77 \pm 10$ & $<0.001^{\mathrm{b}}$ \\
\hline Mild & 3 & 24 & NA & \\
\hline Moderate & 62 & 32 & NA & \\
\hline Severe & 28 & 44 & NA & \\
\hline Profound & 7 & 0 & NA & \\
\hline Hypertensive $^{\mathrm{d}}(\%)$ & 1 & 8 & 0 & N.S \\
\hline $\operatorname{Obesitas}^{\mathrm{e}}(\%)$ & 22 & 20 & 3 & N.S \\
\hline Regulated hypothyroidism (\%) & 30 & 5 & 3 & $<0.05^{\mathrm{a}, \mathrm{c}}$ \\
\hline Regulated hyperthyroidism (\%) & 4 & 0 & 0 & N.S \\
\hline
\end{tabular}


Table 2 Cardiac responses

Intellectual disability

$\begin{array}{lll}\begin{array}{l}\text { Down syndrome } \\ n=96\end{array} & \begin{array}{l}\text { Non-Down-syndrome } \\ n=25\end{array} & \\ n=33\end{array}$

Controls

$p$-value

Rest

Cardiac output (1/min)

Cardiac index $\left(1 / \mathrm{min} / \mathrm{m}^{2}\right)$

$3.7 \pm 0.9$

$5.3 \pm 1.3$

$4.5 \pm 1.0$

$<0.001^{\mathrm{a}, \mathrm{b}, \mathrm{c}}$

$2.3 \pm 0.5$

$2.8 \pm 0.6$

Stroke volume (ml)

$55.1 \pm 13.6$

$68.3 \pm 13.1$

$33.6 \pm 7.5$

$36.4 \pm 5.9$

$\left(\mathrm{ml} / \mathrm{m}^{2}\right)$

Heart rate (beats $/ \mathrm{min}$ )

${ }^{\mathrm{a} C}$ Control versus Down syndrome patients

${ }^{\mathrm{b}}$ Control versus non-Down syndrome patients with intellectual disability

${ }^{\mathrm{c} D o w n}$ syndrome patients versus non-Down syndrome patients with intellectual disability

${ }^{\mathrm{d}}$ Performed in 52 Down syndrome patients and 22 controls, 11 non-Down syndrome patients with intellectual disability

${ }^{\text {e}}$ Performed in 42 Down syndrome patients and 22 controls, 11 non-Down syndrome patients with intellectual disability
Tilting of the legs ${ }^{\mathrm{d}}$

Cardiac output (1/min)

Cardiac index $\left(1 / \mathrm{min} / \mathrm{m}^{2}\right)$

Stroke volume $(\mathrm{ml})$

Indexed stroke volume $\left(\mathrm{ml} / \mathrm{m}^{2}\right)$

Heart rate (beats/min)

After exercise ${ }^{\mathrm{e}}$

Cardiac output (1/min)

Cardiac index $\left(1 / \mathrm{min} / \mathrm{m}^{2}\right)$

Stroke volume (ml)

Indexed stroke volume $\left(\mathrm{ml} / \mathrm{m}^{2}\right)$

Heart rate (beats/min)

$\begin{array}{cccl}68 \pm 12 & 78 \pm 13 & 64 \pm 12 & <0.001^{\mathrm{b}, \mathrm{c}} \\ 4.0 \pm 1.1 & 6.1 \pm 1.5 & 5.0 \pm 0.8 & <0.001^{\mathrm{a}, \mathrm{b}, \mathrm{c}} \\ 2.5 \pm 0.7 & 3.1 \pm 0.7 & 2.7 \pm 0.3 & <0.01^{\mathrm{c}} \\ 58.1 \pm 15.0 & 78.8 \pm 12.5 & 69.9 \pm 10.4 & <0.001^{\mathrm{a}, \mathrm{c}} \\ 35.7 \pm 7.9 & 39.5 \pm 6.2 & 38.0 \pm 5.9 & \mathrm{NS} \\ 70 \pm 11 & 77 \pm 9 & 72 \pm 10 & \mathrm{NS} \\ & & & \\ 4.7 \pm 1.3 & 7.5 \pm 1.7 & 6.8 \pm 1.3 & <0.001^{\mathrm{a}, \mathrm{c}} \\ 2.9 \pm 0.7 & 3.7 \pm 0.8 & 3.7 \pm 0.5 & <0.001^{\mathrm{a}, \mathrm{b}, \mathrm{c}} \\ 61.8 \pm 15.1 & 80.3 \pm 13.3 & 85.5 \pm 14.8 & <0.001^{\mathrm{a}, \mathrm{c}} \\ 37.7 \pm 7.5 & 40.4 \pm 7.7 & 46.3 \pm 6.8 & <0.001^{\mathrm{a}} \\ 77 \pm 10 & 93 \pm 12 & 79 \pm 9 & <0.001^{\mathrm{a}, \mathrm{b}, \mathrm{c}}\end{array}$

were found. In our previous study, we already showed that left ventricular volumes are smaller in patients with an intellectual disability (with and without DS) compared with healthy controls [24]. This indicates that stroke volumes are restricted to the smaller hearts in persons with DS. Furthermore, an expected higher heart rate response to compensate for the
Fig. 1 Stroke volume and heart rate response after exercise. Caption: Rest values correspond only with the subjects who performed the exercise test

\section{HEART RATE TO EXERCISE}

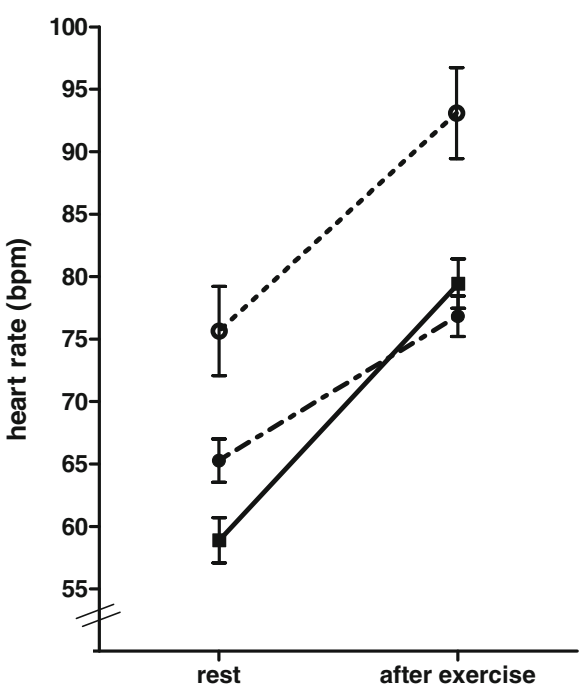

INDEXED STROKE VOLUME TO EXERCISE

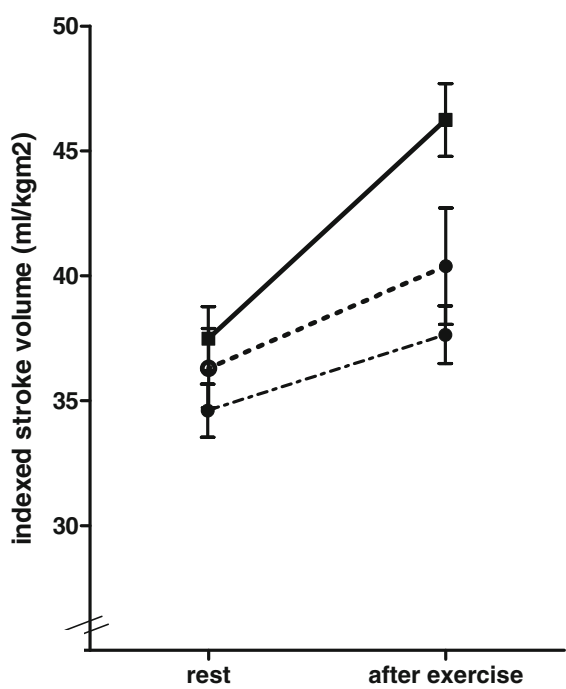

$$
\begin{array}{ll}
\text { Non-Down with } \\
\text { Intellectual disability }
\end{array}
$$


Fig. 2 Cardiac index response. a) Cardiac index response after upright tilting of the legs. b) Cardiac index response after exercise. Caption: Rest values correspond only with the subjects who performed the exercise test
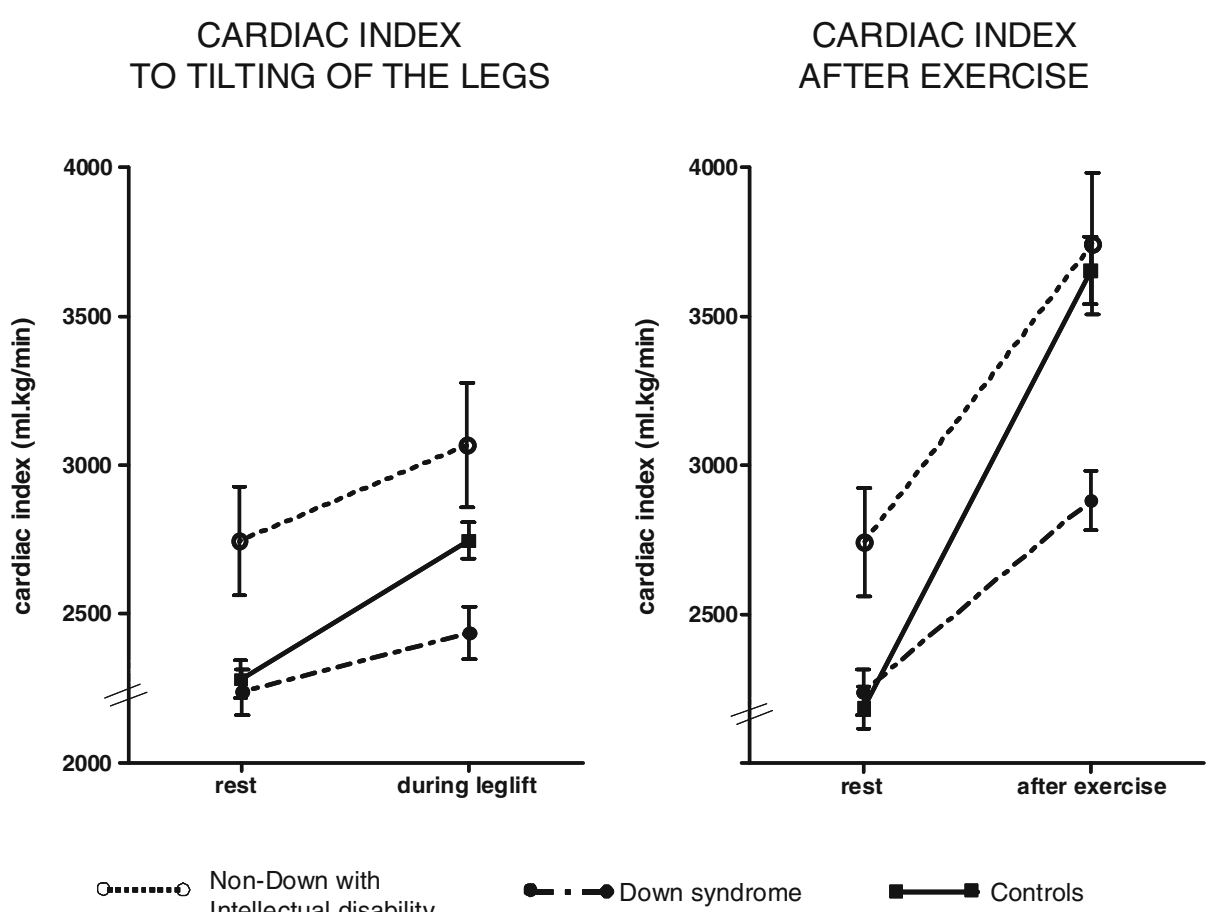

Non-Down with
Intellectual disability

\section{CARDIAC INDEX AFTER EXERCISE}

lower stroke volume was absent in persons with DS. This fits in with the theory of Baynard et al. that peak heart rate is low in individuals with DS [16]. Fernhall et al. suggested that a reduced peak heart rate and low work capacities in individuals with DS could be explained by a diminished catecholamine response to peak exercise [18]. Furthermore, Figueroa et al. reported a reduced heart rate and blood pressure response to sympathetic tasks due to blunted vagal withdrawal and reduced sympathoexcitation [25]. In addition to this reduced sympathicomimetic ability to exercise we demonstrated a reduced stroke volume in adults with DS. The findings in non-DS adults with an intellectual disability show that our results are specific to Down syndrome. In this study, a lower increased heart rate after exercise was found in persons with DS compared with non-DS adults with an intellectual disability. This

Table 3 Multivariate analysis for cardiac index change (rest to exercise)

\begin{tabular}{lrlll}
\hline & $\begin{array}{l}\text { Univariate } \\
\text { Beta }\end{array}$ & $P$ value & $\begin{array}{l}\text { Multivariate } \\
\text { Beta }\end{array}$ & $P$ value \\
\hline Age & -0.21 & 0.07 & -0.24 & 0.009 \\
Male gender & 0.05 & 0.7 & & \\
BMI & -0.07 & 0.6 & & \\
Groups & & & & \\
Controls & 0.59 & $<0.001$ & - & - \\
Down syndrome & -0.57 & $<0.001$ & -0.46 & 0.001 \\
Non Down syndrome & 0.05 & 0.7 & -0.13 & 0.2 \\
$\quad$ with ID & & & & \\
LV end diastolic diameter & 0.62 & $<0.001$ & 0.33 & 0.009 \\
\hline
\end{tabular}

$B M I$ body mass index, $I D$ intellectual disability, $L V$ left ventricular corresponds to the study by Pitetti et al. where individuals without DS with an intellectual disability had a significantly higher mean heart rate than individuals with DS during treadmill walking [26]. Pitetti et al. also found a significantly higher peak VO2 in persons with an intellectual disability without DS [26]. In our study, cardiac index after exercise was similar among healthy controls and non-DS adults with an intellectual disability. Overall, we can conclude that non-DS adults with an intellectual disability are capable of increasing their cardiac capacity adequately, unlike individuals with DS.

A limitation of this study is that exercise testing failed in more than half of the included subjects with an intellectual disability. As expected most subjects with a severe intellectual disability were not able to perform the test due to comorbid physical problems or non-cooperation. However, all other important baseline characteristics were not statistically different from patients with or without successful exercise testing. Secondly, a familiarisation protocol was not carried out. However, by using a relatively simple task, which most adults with an intellectual disability could understand, we minimised possible result errors by inadequate performance of the task. Thereby, patients with inadequate testing (task duration of more than $45 \mathrm{~s}$ ) were excluded for analysis of cardiac output change.

\section{Conclusion}

Cardiac response to exercise is diminished in adults with DS. Stroke volumes were significantly lower in DS during exercise and a compensated increased heart rate was absent. 
An adequate cardiac response seems to exist in non-DS adults with an intellectual disability. A reduced cardiac response could be clinically relevant when considering that cardiac reserve may be inadequate in situations where higher cardiac output is needed, for example during fever and for sport activities. Also the effect of training programs to increase exercise capacity in the DS population could be negatively influenced when adults with DS are less able to increase their cardiac index while exercising.

Acknowledgements This work was supported by Actelion Pharmaceuticals Nederland B.V. Actelion Pharmaceuticals Nederland B.V provided material support (portable General Electric VIVID I) for this research. The sponsor had no role in the design and conduct of the study; collection, management, analysis, and interpretation of the data; and preparation, review, or approval of the manuscript.

Open Access This article is distributed under the terms of the Creative Commons Attribution License which permits any use, distribution, and reproduction in any medium, provided the original author(s) and the source are credited.

\section{References}

1. Bittles AH, Bower C, Hussain R, et al. The four ages of Down syndrome. Eur J Publ Health. 2007;17:221-5.

2. de Graaf G, Vis JC, Haverman M, et al Assessment of prevalence of persons with Down syndrome: a theory-based demographic model. JARID 2010

3. Finesilver C. A new age for childhood diseases. Down syndrome. RN. 2002;65:43-8.

4. Glasson EJ, Sullivan SG, Hussain R, et al. The changing survival profile of people with Down's syndrome: implications for genetic counselling. Clin Genet. 2002;62:390-3.

5. Duffels MG, Vis JC, van Loon RL, et al. Down patients with Eisenmenger syndrome: is bosentan treatment an option? Int $\mathrm{J}$ Cardiol. 2009; 134:378-83.

6. Duffels MG, Vis JC, van Loon RL, et al. Effect of bosentan on exercise capacity and quality of life in adults with pulmonary arterial hypertension associated with congenital heart disease with and without Down's syndrome. Am J Cardiol. 2009;103:1309-15.

7. Barnhart RC, Connolly B. Aging and Down syndrome: implications for physical therapy. Phys Ther. 2007;87:1399-406.

8. Bell AJ, Bhate MS. Prevalence of overweight and obesity in Down's syndrome and other mentally handicapped adults living in the community. J Intel Disabil Res. 1992;36(Pt 4):359-64.

9. Prasher VP, Filer A. Behavioural disturbance in people with Down's syndrome and dementia. J Intel Disabil Res. 1995;39(Pt 5):432-6.

10. Shamas-Ud-Din S. Genetics of Down's syndrome and Alzheimer's disease. Br J Psychiatry. 2002;181:167-8.
11. Vis JC, Duffels MG, Winter MM, et al. Down syndrome: a cardiovascular perspective. J Intellect Disabil Res. 2009;53:41925.

12. Gonzalez-Aguero A, Vicente-Rodriguez G, Moreno LA et al. Health-related physical fitness in children and adolescents with Down syndrome and response to training. Scand J Med Sci Sports 2010

13. Fernhall B, Pitetti KH, Rimmer JH, et al. Cardiorespiratory capacity of individuals with mental retardation including Down syndrome. Med Sci Sports Exerc. 1996;28:366-71.

14. Fernhall B, McCubbin JA, Pitetti KH, et al. Prediction of maximal heart rate in individuals with mental retardation. Med Sci Sports Exerc. 2001;33:1655-60.

15. Baynard T, Pitetti KH, Guerra M, et al. Age-related changes in aerobic capacity in individuals with mental retardation: a $20-\mathrm{yr}$ review. Med Sci Sports Exerc. 2008;40:1984-9.

16. Baynard T, Pitetti $\mathrm{KH}$, Guerra $\mathrm{M}$, et al. Heart rate variability at rest and during exercise in persons with Down syndrome. Arch Phys Med Rehabil. 2004;85:1285-90.

17. Guerra M, Llorens N, Fernhall B. Chronotropic incompetence in persons with Down syndrome. Arch Phys Med Rehabil. 2003;84:1604-8.

18. Fernhall B, Baynard T, Collier SR, et al. Catecholamine response to maximal exercise in persons with Down syndrome. Am J Cardiol. 2009;103:724-6.

19. Millar AL, Fernhall B, Burkett LN. Effects of aerobic training in adolescents with Down syndrome. Med Sci Sports Exerc. $1993 ; 25: 270-4$.

20. Varela AM, Sardinha LB, Pitetti KH. Effects of an aerobic rowing training regimen in young adults with Down syndrome. Am J Ment Retard. 2001;106:135-44.

21. Tsimaras V, Giagazoglou P, Fotiadou E, et al. Jog-walk training in cardiorespiratory fitness of adults with Down syndrome. Percept Mot Skills. 2003;96:1239-51.

22. Schiller NB, Shah PM, Crawford M, et al. Recommendations for quantitation of the left ventricle by two-dimensional echocardiography. American Society of Echocardiography Committee on Standards, Subcommittee on Quantitation of Two-Dimensional Echocardiograms. J Am Soc Echocardiogr. 1989;2:358-67.

23. Lang RM, Bierig M, Devereux RB, et al. Recommendations for chamber quantification: a report from the American Society of Echocardiography's Guidelines and Standards Committee and the Chamber Quantification Writing Group, developed in conjunction with the European Association of Echocardiography, a branch of the European Society of Cardiology. J Am Soc Echocardiogr. 2005;18:1440-63.

24. Vis JC, de Bruin-Bon RH, Bouma BJ et al. 'The sedentary heart': physical inactivity is associated with cardiac atrophy in adults with an intellectual disability. Int J Cardiol. 2011.

25. Figueroa A, Collier SR, Baynard T, et al. Impaired vagal modulation of heart rate in individuals with Down syndrome. Clin Auton Res. 2005; $15: 45-50$.

26. Pitetti KH, Climstein M, Campbell KD, et al. The cardiovascular capacities of adults with Down syndrome: a comparative study. Med Sci Sports Exerc. 1992;24:13-9. 